\title{
Characterization of kaolin flocs formed by polyacrylamide as flocculation aids
}

\author{
Zhe Zhu ${ }^{\text {a,b }}$, Tao $\mathrm{Li}^{\mathrm{b}, *}$, Jiajuan Lu ${ }^{\mathrm{b}}$, Dongsheng Wang ${ }^{\mathrm{b}}$, Chonghua Yao ${ }^{\mathrm{a}}$ \\ a Resources and Environmental Engineering Institute, East China University of Science and Technology, Shanghai 200237, PR China \\ b State Key Laboratory of Environmental Aquatic Chemistry, Research Center for Eco-Environmental Sciences, Chinese Academy of Sciences, Beijing 100085, PR China
}

\section{A R T I C L E I N F O}

\section{Article history:}

Received 3 April 2007

Received in revised form 19 January 2009

Accepted 25 January 2009

Available online 1 February 2009

\section{Keywords:}

Charge density

Molecular weight

Size

Fractal dimension

Strength

Reversibility

\begin{abstract}
A B S T R A C T
Although the synthetic organic polymers have been widely applied as flocculant aids to improve solid-liquid separation efficiency, it is not very clear how the charge type and molecular weight of the polymers influence the characteristics of flocs. In this paper, floc characteristics, such as floc size, density, structure, strength and reversibility were investigated in details in terms of their response to the polyacrylamide (PAM) with different charge types and molecular weights. Laser light scattering, image analysis and settling technology were employed simultaneously. The results showed that floc size induced by anionic PAM with medium molecular weight (A130) and ultra high molecular weight (A115) was $419 \mu \mathrm{m}$ and $438 \mu \mathrm{m}$ respectively, correspondingly, floc size induced by cationic PAM with medium molecular weight (C448) and very high molecular weight (C498) was $731 \mu \mathrm{m}$ and $796 \mu \mathrm{m}$. Meanwhile, floc fractal dimension was inversely proportional to polymer molecular weight and the values were between 1.91 and 2.40 . In addition, floc density was affected by molecular weight of anionic PAM more dramatically than that of cationic PAM. For a given floc size, the density increased with molecular weight. Furthermore, flocs formed by cationic PAM showed much greater shear-resistant ability and reversibility than anionic PAM. Nevertheless, the poor reversibility of the flocs formed by high molecular weight was also observed. An explanation for the resultant floc characteristics was offered in terms of flocculation mechanisms.
\end{abstract}

(c) 2009 Elsevier B.V. All rights reserved.

\section{Introduction}

Floc characteristics, such as size, structure, density, strength, play an important role in determining the efficiency of many industrial separation processes (Zhou and Franks, 2006). In the previous research, characteristics of flocs formed in the coagulation process by metal salts have been well understood (Jarvis et al., 2005a; Francois, 1987; Spicer and Pratsinis, 1996; Chakraborti et al., 2000). The accurate estimation of flocs characteristics is valuable for evaluating water treatment performance under certain coagulation conditions.

Synthetic organic polymers, such as polyacrylamide (PAM) and polydimenthydiallyammomium chloride (PDMDAAC), have been widely applied as flocculant aids in many industries, including thickening operations in mineral processing, industrial tailings dewatering, papermaking and water treatment (Yan et al., 2004). The use of polymers in water treatment can achieve rapid flocculation and result in better settling or filtrating behaviors. Several studies have demonstrated that various flocculation mechanisms are responsible for different floc characteristics (Li et al., 2006). It has been established that the polymers can flocculate particles via bridging, charge neutralization, electrostatic patch and depletion flocculation

\footnotetext{
* Corresponding author. Tel.: +86 10 62849144; fax: +861062849138. E-mail address: taoli@rcees.ac.cn (T. Li).
}

mechanisms. Meanwhile, polymer properties, such as molecular weight, charge density and charge types, affect flocculating and destabilizing actions (Zhou and Franks, 2006; Glover et al., 2001). Generally, bridging flocculation is usually occurred by adding polymer with high molecular weight to a dispersion of colloidal particles. One obvious consequence of bridging flocculation is that the flocs produced can be much larger and stronger than when particles are destabilized by simply reducing the repulsion between them (usually by adding melt salts) (Ray and Hogg, 1987). Yukselen and Gregory (2004) considered higher molecular weight polymers should give stronger flocs as a result of polymer bridging. While, bridging flocs usually display loosely bound structures due to their large size. Glover et al. (2001) gave the values of mass fractal dimension, which varied from 2.29 to 2.33, for alumino-PAA flocs under low shear conditions. These low values indicate that flocs produced via bridging mechanism have relatively loose structures. When low molecular weight polymers tend to adsorb and neutralize the opposite charges on the particles, charge neutralization becomes a major mechanism in polymer-particle interactions. Yu et al. (2006) investigated the flocculation of kaolin particles by polyelectrolytes and found that the floc structures via charge neutralization are more compact. Additionally, electrostatic patch model applies when the particles have a fairly low density of immobile surface charges and the adsorbing polymers have high charge density. The particles may have "patches" of adsorbed polymer and these patches are able to 
contact with bare surface, but oppositely charged surface on another particle, giving quite strong attachment (Ye et al., 2007). Gregory (1997) thought that although the electrostatic patch produces flocs that are stronger than those obtained from simple charge neutralization, these flocs are generally not as strong as those produced by the bridging process.

Previous research on the behaviors of adsorption and flocculation of particles had mainly focused on the complex effects of flocculation conditions on the removal efficiency, and thus, most of the experimental results were principally limited in the residual turbidity (Leu and Ghosh, 1988; Fan et al., 2000; Yu and Somasundaran, 1996), few investigations pay attention to distinction of floc itself. Knowledge of floc characteristics is essential for the optimization of flocculation process (Glover et al., 2001; Ray and Hogg, 1987; Yukselen and Gregory, 2004; Yu et al., 2006). Nasser and James (2006) have investigated the effect of charge density and molecular weight of PAM on the flocculation and settling behaviors of kaolin suspensions. They demonstrated that the floc size and settling rate show a strong dependence on polymer charge type. Meanwhile, they have considered that the difference in the compression sensitivity of the flocculated slurries might be attributed to floc structure-related adsorption. However, as flocs are considered as fractal matters, the information is limited, particularly, for organic polymer used as flocculant aids. Better understanding of the influence of polymer properties on the floc physical characteristics may lead to better choice of flocculant aids for specific use. In this study, three typical analysis methods, such as laser light scattering, image analysis and settling technology were employed to obtain the information about floc characteristics. The overall objective of this work is to systematically examine the physical characteristics of kaolin flocs coagulated by aluminum sulfate in combination with PAM used as flocculants aids with different molecular weights and charge types.

\section{Background theories}

\subsection{Fractal dimension}

Aggregates of colloidal particles exhibit fractal structure (Gregory, 1997; Jiang and Logan, 1991; Johnson et al., 1996). Fractal theory provides a quantitative means to describe the degree to which primary particles fill the space within the nominal volume occupied by an aggregate. If an aggregate has a fractal structure, the relationship between the aggregate mass $(M)$ and linear measure of its size $(R)$ is simply

$M(R) \propto R^{D_{\mathrm{F}}}$

where $D_{\mathrm{F}}$ is the mass fractal dimension and can have a value between 1 and 3 (Bushell et al., 2002). High $D_{\mathrm{F}}$ means the primary particles in an aggregate are arranged compactly, while low $D_{\mathrm{F}}$ results from large, highly branched and loosely bound structures. According to previous research, methods utilized to characterize the fractal dimension are mainly microscopy, light scattering, and settling techniques (Wu et al., 2002; Koen and Willy, 1997; Chakraborti et al., 2003; Tang et al., 2002).

\subsection{Floc density}

The density $(\rho(R))$ of a fractal aggregate is estimated by the mass of the aggregate divided by the volume enclosing the aggregate, which leads to the following expression:

$\rho(R) \propto R^{D_{\mathrm{F}}-3}$

This relationship implies that a very important consequence of fractal aggregates is that their density decreases appreciably as the size increases (Tambo and Watanabe, 1979; Zhao, 2004). Settling technique is an effective means to estimate the floc density. The method for floc density calculation by settling technique was described by Glover et al. (2000).

\subsection{Floc strength}

Besides size, fractal dimension and density, floc strength is another important operational parameter in the solid-liquid separation process. Floc strength is a function of inter-particle forces holding the particles together and it depends on both the strength of the interparticle bonds between the components of the aggregate and the number of bonds (Bache et al., 1997). Therefore, floc will be broken if the stress applied at its surface is larger than the bonding strength within the floc (Boller and Blaser, 1998). Floc strength is hard to characterize due to the inherent complexity, fragility and variation in floc size, shape and composition. In earlier study, floc strength was defined from the empirical relationship between the size of the surviving aggregates and the applied shear that flocs were subjected to during turbulent flow (Tambo and Hozumi, 1979). As the shear rate is increased, the maximum floc size decreases and this remains one of the most effective empirical indications of floc strength. The larger the flocs formed under given shear conditions, the stronger they are. Therefore, many researchers defined strength factor to indicate floc strength (Jarvis et al., 2005b). Other researchers estimated the floc strength by micro mechanical measurement of the floc bonding strength via its rupture force (Yeung and Pelton, 1996).

\section{Materials and methods}

\subsection{Suspension}

Kaolin was applied as a model suspension. The stock suspension of kaolin was prepared in deionized water, which was similar to Li et al. (2007). The solid concentration of suspension was determined by gravimetrically to be $137 \mathrm{~g} / \mathrm{L}$. The average size of the particles in suspension was close to $5 \mu \mathrm{m}$, measured by a laser diffraction instrument (Mastersizer 2000, Malvern, UK).

\subsection{Coagulant and flocculants}

Aluminum sulfate $\left(\mathrm{Al}_{2}\left(\mathrm{SO}_{4}\right)_{3} \cdot 18 \mathrm{H}_{2} \mathrm{O}\right.$, analytic reagent) was used as coagulant. Stock alum solution was prepared as $0.1 \mathrm{M}$ solution in deionized water. Four commercial PAMs with different charge types and molecular weights supplied by Cytec Industries (USA) were involved as flocculant aids. All polymers were obtained in powder form and stock solution with a concentration of $0.1 \%$ was prepared for each polymer prior to use. Some properties of these polymers are listed in Table 1.

\subsection{Methods}

\subsubsection{Determination of optimum polymer dose}

Optimal polymer dose was determined by carrying out a series of jar tests with polymer dose increment. Coagulation-flocculation experiments were carried out by variable speed jar tester with $50 \times 40 \mathrm{~mm}$ flat paddle impellers and $1 \mathrm{~L}$ cylindrical beakers containing $800 \mathrm{~mL}$ sample suspension. The stock suspension was diluted in tap water to give a

Table 1

Specification of the polyacrylamide as a flocculant aids used in the experiment.

\begin{tabular}{llll}
\hline Polymer & Charge density (\%) & Molecular weight & Charge type \\
\hline A130 & 20 & Medium & Anionic \\
A115 & 20 & Ultra high & Anionic \\
C448 & 55 & Medium & Cationic \\
C498 & 55 & Very high & Cationic \\
\hline
\end{tabular}


kaolin concentration of $100 \mathrm{mg} / \mathrm{L}$. The initial solution $\mathrm{pH}$ was kept around $7.80 \pm 0.05$ (MP220 Mettler-Toledo, Switzerland). Rapid mixing at $200 \mathrm{rpm}$ for $1 \mathrm{~min}$, followed by slow stirred at $40 \mathrm{rpm}$ for $10 \mathrm{~min}$, finally, settling for $15 \mathrm{~min}$. At the beginning of rapid mixing, $0.1 \mathrm{mM}$ aluminum sulfate solution was added to the beaker. Zeta potential was measured by Zetasizer 2000 (Malvern, UK) after alum dosing. Then, PAM was dosed after $30 \mathrm{~s}$ rapid mixing. After $15 \mathrm{~min}$ settling, $30 \mathrm{~mL}$ sample was withdrawn from about $20 \mathrm{~mm}$ below the top supernatant for residual turbidity measurement (Turbidimeter 2100N, Hach, USA). And then the optimal polymer dose was determined based on the minimum residual turbidity. All the subsequent experiments were conducted at the optimal polymer dose.

\subsubsection{Floc size and fractal dimension}

The size and fractal dimension of the aggregates formed at the optimal flocculation condition were measured by using a laser diffraction instrument (Malvern Mastersizer 2000, Malvern, U.K.). The suspension was circulated by drawing water through the optical unit of the Mastersizer and back into the beaker by a peristaltic pump with plastic tube of $5 \mathrm{~mm}$ inner diameter at a flow rate of $25 \mathrm{~mL} / \mathrm{min}$. Size measurements were taken every $40 \mathrm{~s}$ for the duration of the jar tests. The average size of flocs given by Malvern Mastersizer 2000 is in terms of the median equivalent volumetric diameter $D_{[4,3]}$, assuming that flocs are solid objects of spherical shape. Normally, floc structure has been determined quantitatively by the mass fractal dimension $D_{\mathrm{F}}$. In this study, mass fractal dimension is measured by light scattering technology. The method is briefly given as follows: the scattered intensity $I$ as a function of the magnitude of the scattering wave vector $Q$ is measured, where,

$Q=\frac{4 \pi n \sin (\theta / 2)}{\lambda}$

$n, \theta$, and $\lambda$ are the refractive index of the medium, the scattered angle, and the wavelength of radiation in vacuum, respectively. For independently scattering aggregates, $I$ is related to $Q$ and the fractal dimension $D_{\mathrm{F}}$.

$I \propto Q^{-D_{\mathrm{F}}}$

So, on a log-log scale if there is a straight line, the slope of which is $D_{\mathrm{F}}$.

Mastersizer 2000 was employed to determine the scattered light intensity as a function of the scattered angle.

\subsubsection{Floc density}

Floc density was determined by settling experiment which had been reported and the experiment devices in this investigation were similar with others (Glover et al., 2000). Briefly, after slow mixing for $10 \mathrm{~min}$, about $2 \mathrm{~mL}$ samples of flocs were transferred by using a wide mouthed pipette and carefully released into the $30 \mathrm{~mm} \times 30 \mathrm{~mm} \times 500 \mathrm{~mm}$ (Length, Width and Height) settling column which was surrounded by a water jacket and was maintained at $20^{\circ} \mathrm{C}$. Movement of individual floc in the settling column was viewed by a CCD (Charge-Couple Devices) camera (Watec, Japan) equipped with a microscope Len (Computar, Japan). The column was illuminated by a fiber optic light source. Image analysis software (Mivnt, DaHeng, China) was applied to determine the floc settling velocity. The calculations involved floc density and equivalent sphere diameter were introduced by Glover et al. (2000).

\subsubsection{Floc breakage and recovery}

In order to investigate the floc breakage and regrowth, the following procedures were conducted. The rapid mixing at $200 \mathrm{rpm}$ for $1 \mathrm{~min}$, followed by floc growth phase at $40 \mathrm{rpm}$ for $10 \mathrm{~min}$, and then followed by a breakage phase at $400 \mathrm{rpm}$ for $3 \mathrm{~min}$. After breakage, the other slow mixing at $40 \mathrm{rpm}$ was reintroduced for a further $10 \mathrm{~min}$. Likewise, Mastersizer was also utilized to monitor the floc breakage and regrowth process. The relative breakage and regrowth degree of different flocculated systems were compared by application of floc strength and recovery factors which may be calculated as follows:

Strength factor $=\frac{d_{(2)}}{d_{(1)}} \times 100$

Recovery factor $=\frac{d_{(3)}-d_{(2)}}{d_{(1)}-d_{(2)}} \times 100$

where $d_{(1)}$ is the average floc size of the plateau before breakage, $d_{(2)}$ is the floc size after the floc breakage, and $d_{(3)}$ is the floc size after regrowth to the new plateau. An increased value of strength factor indicates that flocs are better able to withstand shear force and thus should be considered stronger than those with a lower factor. Meanwhile, an increase in the recovery factor shows that flocs have better regrowth ability after high shear (Jarvis et al., 2005c).

\section{Results and discussion}

\subsection{Flocculant aids dosing}

The zeta potential of original kaolin suspension was about $-17.1 \mathrm{mV}$ and the turbidity was close to 71.2 NTU. When the primary coagulant, alum $(0.1 \mathrm{mM}$ as $\mathrm{Al})$, was added, the zeta potential increased to $-4.2 \mathrm{mV}$. The dosage chosen for all polymers was $125 \mu \mathrm{g} / \mathrm{L}$, which was determined by the lowest residual turbidity range appearing, within these polymer doses.

\subsection{The effect of charge type and molecular weight of PAM on the floc size}

Floc size was quantified by median equivalent volumetric diameter $D_{[4,3]}$. In Fig. 1, floc size induced by anionic PAM with A130 and A115 was $419 \mu \mathrm{m}$ and $438 \mu \mathrm{m}$ individually, while, floc size induced by cationic PAM with C448 and C498 was $731 \mu \mathrm{m}$ and $796 \mu \mathrm{m}$. It showed that floc size increased with polymer molecular weight. When the PAM with high molecular weight was added, the polymer long chain extending to the particle surface might be able to span over the electrical double layers of the approaching particles and cause bridging flocculation to occur. Meanwhile, an increase in polymer molecular weight results in an increase in attachment between polymer and particles, and then the adsorption of the polymer on the particle surface is enhanced and thus facilitates bridging. On the other hand, bridging flocculation occurs when the polymer has an expanded configuration. As the polymer molecular weight is higher, reconformation of the long chain at the particle surface occurs more slowly. It is

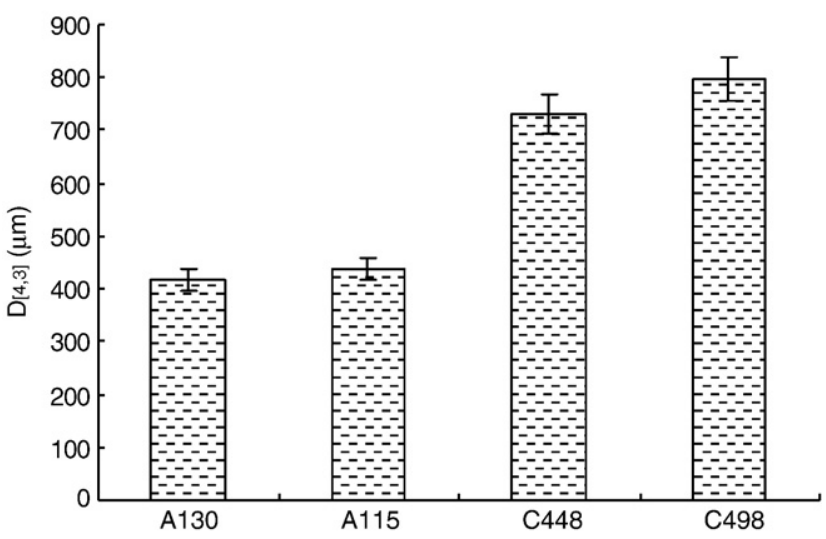

Fig. 1. Effect of charge type and molecular weight on the size of flocs. 
likely that another particle may hit the surface before the relaxation of the polymer to the particle surface has occurred. In this case, bridging flocculation occurs. In summary, no matter what charge type the PAM is, the polymer with high molecular weight is favorable for the formation of aggregate with large size. Glover et al. (2001) draw a similar conclusion when they used polyacrylic acid to investigate the effect of molecular weight on the floc size. The result indicates a move toward bridging flocculation mechanism for the higher molecular weight polymers as it is commonly observed that bridging flocculation results in flocs of large size.

Fig. 1 also showed that the flocs aggregated by adding cationic PAM were larger than those formed by anionic PAM. It could be ascribed to the bonding between the cationic polymer and negative edges of the kaolin-alum particles or aggregates via electrostatic attraction. It has to be pointed out that the charge of kaolin particles firstly coagulated by alum was still negative and the zeta potential was $-4.2 \mathrm{mV}$. When the aluminum sulfate was added to the kaolin suspension as the primary coagulant, small flocs were formed. In this process, the main coagulation mechanism could be charge neutralization, and no obvious aluminum hydroxide formed under this relatively low Al dose. Thereafter, cationic PAM, added as the flocculant aid, was adsorbed on the particle surface by cationic group. Accordingly, zeta potential had been increased from -4.2 to $-1.9 \mathrm{mV}$ for C448 and to $-0.5 \mathrm{mV}$ for C498, respectively. That is to say, the negative charge of particle or floc surface was further neutralized. The amount of polymer required to neutralize the particle charge is inversely proportional to charge density and the high charge density polymers neutralize the surface charge of particle more rapidly than the low charge density polymers. At the concentration of $125 \mu \mathrm{g} / \mathrm{L}$, both C448 and C498 with the same charge density showed similar ability of the polymers to neutralize the particles' surface charge. Moreover, the bridge length was shortened due to the reducing of repulsive interaction between the particles, which favored bridging flocculation. On the contrary, when the anionic PAM was added, only relatively small flocs were allowed to be formed due to the electrostatic repulsion. In this case, if electric double layer repulsion was spanned by anionic PAM, very high molecular weight would be needed. In conclusion, cationic PAM can lead to a larger floc due to the contribution of both charge neutralization and bridging.

\subsection{The effect of charge type and molecular weight of PAM on the fractal} dimension

As shown in Fig. 2, the floc fractal dimension decreased with increasing polymer molecular weight and the values were in the

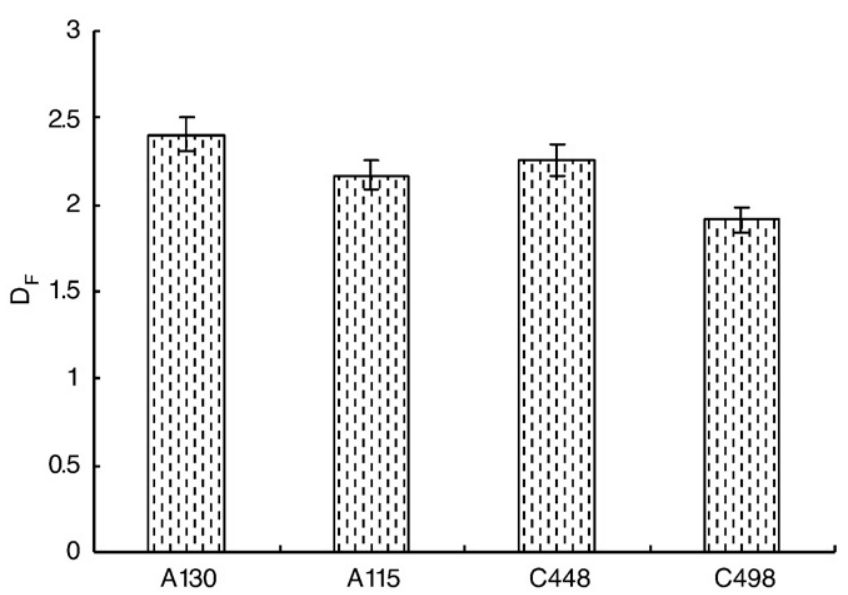

Fig. 2. Effect of charge type and molecular weight on the fractal dimension of flocs. (a)

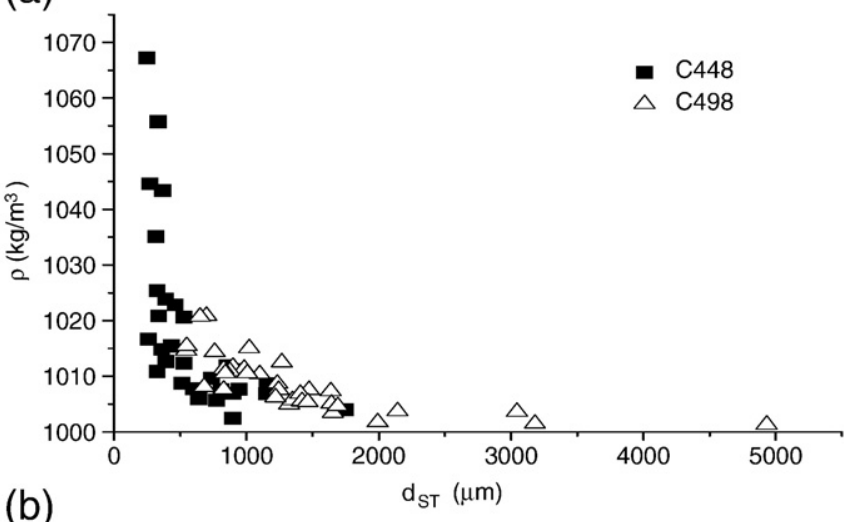

(b)

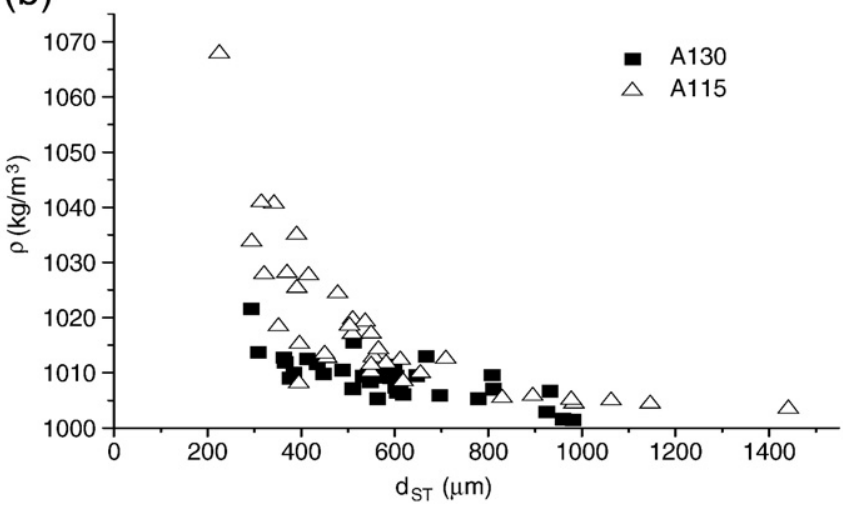

Fig. 3. Effect of charge type and molecular weight on the density of flocs.

region of 1.91 and 2.40. For cationic polymer with relatively low molecular weight, the conformation of the polymer molecule is similar to the suspended particle, and thus, the charge neutralization became a dominative mechanism. Meanwhile, cationic polymer tended to flatten onto the negative kaolin or small flocs surfaces and led to densely packed floc structure. As the polymer molecular weight was relatively high, bridging would be invoked due to the long chain length. Consequently, as the flocculation mechanism moves toward a case of bridging, aggregates with open structure are formed. For anionic PAM, the electrostatic repulsion between kaolin particles and the anionic molecular chains allows flocculation to occur mainly via bridging mechanism. Therefore, the polymer molecule interacts with particles at long distance arising from the electrostatic repulsion. These repulsive forces allow the polymer molecules to be extended and produce loops and tails, which lead to the formation of open structures flocs. Due to the fact that floc has a fractal characteristic, for the polymer with higher molecular weight, the formation of loosely bound floc structure is a result to larger floc size.

It was also found from Fig. 2 that the cationic PAM gave an overall lower fractal dimension than anionic PAM, which appears to be inconsistent with the flocculation mechanisms. Actually, this behavior is simply a reflection of the fact that lower fractal dimension for cationic PAM is an inevitable consequence of the larger size, if the flocs have fractal character.

\subsection{The effect of charge type and molecular weight of PAM on the floc density}

The measured size-density relationship has been shown in Fig. 3. Floc density formed by dosing flocculant aids were varied from $1001 \mathrm{~kg} / \mathrm{m}^{3}$ to $1070 \mathrm{~kg} / \mathrm{m}^{3}$. As shown, floc density decreased appreciably as the size increased, roughly in accordance with a power law relationship between density and size reported by Tambo 
(a)

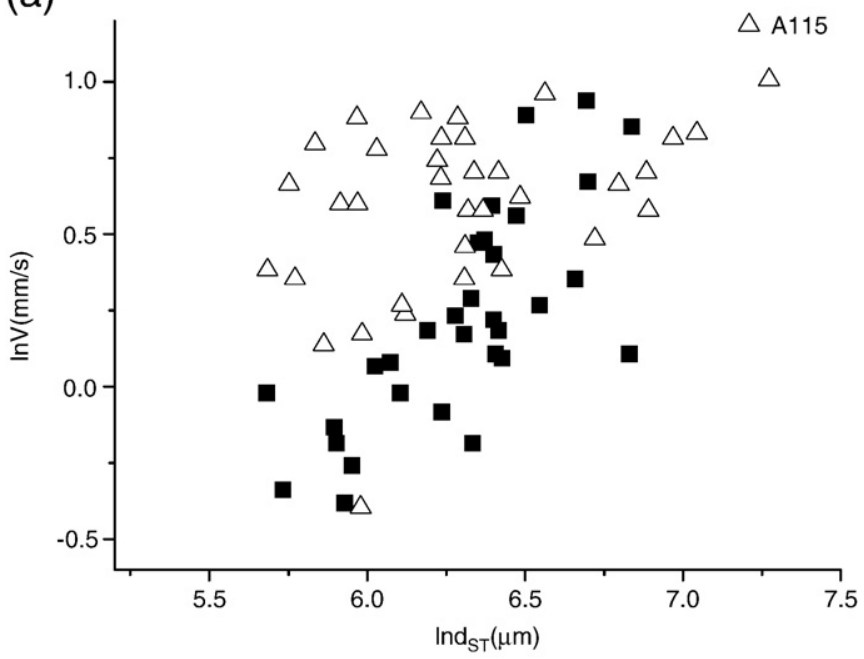

(b)

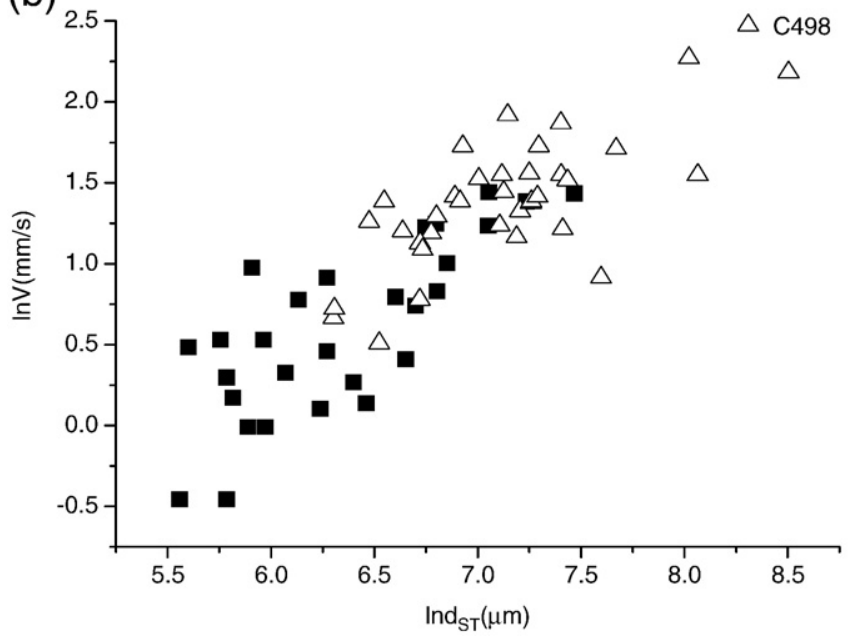

Fig. 4. Effect of charge type and molecular weight on the settling velocities of flocs.

and Watanabe (1979). Floc density was affected by molecular weight dramatically when using anionic PAM. For a given floc size, the density increased with molecular weight. As comparing, in the case of cationic PAM applying, there was no obvious distinction among floc density with various molecular weights. The higher molecular weight of anionic PAM has much stronger affinity for adsorption on the particle surfaces attributing to having more active groups. While, in the case of cationic PAM, both charge neutralization and bridging promote the aggregation of flocs. Because not only molecular weight but also charge density dominates the flocculation process, the contribution from molecular weight is less important.

4.5. The effect of charge type and molecular weight of PAM on the floc settling

The size-settling velocity relationship was plotted on the logarithmic curve as shown in Fig. 4. The floc settling velocity increased with floc size for both anionic and cationic PAM and the values were between $0.6 \mathrm{~mm} / \mathrm{s}$ and $9.7 \mathrm{~mm} / \mathrm{s}$. In addition, the settling rates of the same sized flocs for the polymer with higher molecular weight were larger than those with lower molecular weight. As mentioned above, the bridging flocculation enhanced by the higher molecular weight polymer was further supported by the settling velocity improvement.
Table 2

Strength factor and recovery factor of kaolin flocs induced by anionic PAM and cationic PAM with different molecular weight.

\begin{tabular}{lll}
\hline Polymer & Strength factor (\%) & Recovery factor (\%) \\
\hline A130 & 22 & 33 \\
A115 & 27 & 32 \\
C448 & 26 & 54 \\
C498 & 40 & 51 \\
\hline
\end{tabular}

4.6. The effect of charge type and molecular weight of PAM on the floc breakage and regrowth

As presented in Table 2 and Fig. 5, flocs induced by C448 and C498 were better able to resist shearing condition and their strength factor values were $26 \%$ and $40 \%$, individually, meanwhile, these were higher than the values of $22 \%$ for $A 130$ and $27 \%$ for A115. Therefore, at a given molecular weight, flocs formed by cationic PAM showed much greater shear-resistant ability than those of anionic PAM. Moreover, at the same charge type, higher molecular weight PAM gave stronger flocs.

As we know, floc strength is closely related to the particle bond strength and the number of interparticle bonds. Electrostatic attraction between the positively charged polymer and negative surface charge of the particle gives rise to stronger bonds than those caused by negatively charged polymer. On the other hand, the repulsion force between anionic polymer and negative particle surfaces allows for further expansion of the polymer conformation, leading to a looser floc structure and which tends to be weaker and less resistant to break up. For the same charge type, the floc strength induced by higher

(a)
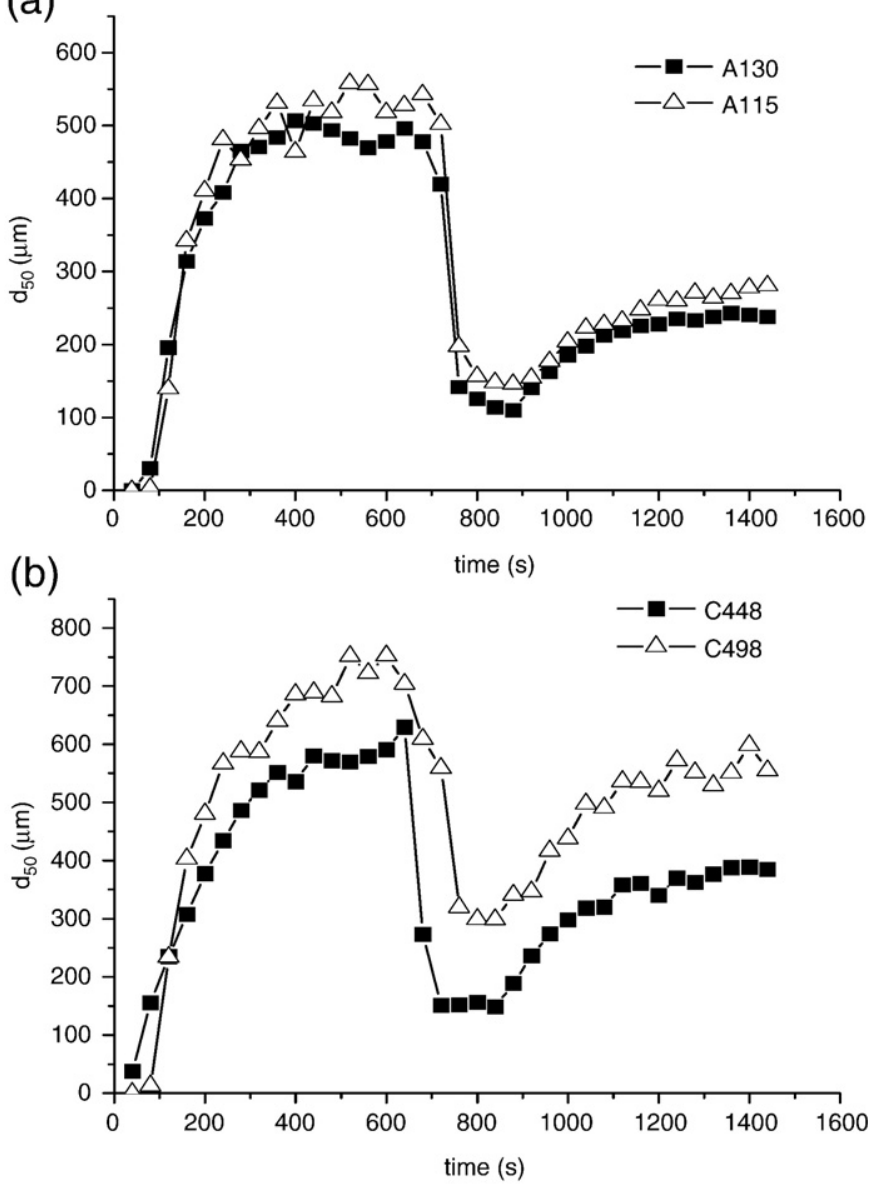

Fig. 5. Flocs breakage and reformation by using PAMs with different charge types and molecular weights. 
molecular weight polymer is stronger than those of lower molecular weight. It can be attributed to stronger bridge formed by larger polymer molecule between two particles and which contains more locations for contacting, and thus, more force is required to break them apart.

It was also evident from Table 2 that the two charge type PAMs showed significant difference in recovery factor. The regrowth ability of flocs induced by anionic PAM was lower than that of cationic PAM. This is due to the difference in flocculation mechanism. Anionic PAM flocculated fine particles mainly by bridging mechanism. High shear rates may cause polymer tails and loops broken and floc fragmentation will appear. Thus rearrangement of the residual polymer on the particles surfaces after breakage may occur, tending to flatten the polymer chains onto the particle surface and reducing their ability to form polymer bridges between particles. However, cationic PAM flocculated fine particles by charge neutralization and bridging mechanism simultaneously. As the flocs are broken by exposure to high hydrodynamic shear, the bridging polymer may undergo scission. When the turbulence is lowered, the adsorbed polymer may be in reconformation on the particle surface, leading to domain with localized excess charge of polymer and form positive patches. Direct electrostatic attraction between the particles is thus promoted and domains of opposite charge on adjacent particles interact, giving strong attachment to form flocs. As a result, cationic PAM showed better reflocculation ability. Yoon and Deng (2004) considered that the initial flocculation ability of the polymer is closely related to the molecular weight, but the reflocculation ability is more dominated by the charge density of the polymer.

Additionally, the relationship between molecular weight and reflocculation ability was shown in Table 2. It has been well known that as the polymer molecular weight is increased there is a tendency for the flocculation mechanism to move toward bridging flocculation. Once bridging polymer is subjected to scission and chemical bonds are broken, there is no likely that particles can reflocculate again. According to these results, comparing with charge density of polymer, the molecular weight has a negative effect on floc reversibility and which is consistent with Negro et al. (2005).

\section{Conclusions}

The effects of molecular weight and charge type of PAM on characteristics of kaolin flocs were investigated. An increase in polymer molecular weight increases the floc size as the long molecular chain can interact with particles at longer distances and thus leads to a looser floc structure with larger size. Meanwhile, cationic PAM can form flocs with larger size than anionic PAM due to the contribution of both charge neutralization and bridging. Floc density was affected by molecular weight dramatically when using anionic PAM. For a given floc size, the density increased with molecular weight. As comparing, in the case of cationic PAM applying, there was no obvious distinction among floc density with various molecular weights. In addition, because of the electrostatic attraction between the positively charged polymer and negative surface of the particle, cationic PAM shows much greater shear-resistant ability and reversibility than anionic PAM. As the polymer molecular weight is increased, polymer chain is prone to scission by exposure to high hydrodynamic shear. Therefore, higher molecular weight PAM gives flocs with poorer reversibility. Summarily, in order to obtain favorable solid-liquid separation for specific use, it is necessary to carefully select the proper charge type and molecular weight of polymer and pay more attention to the effects of floc characteristics.

\section{Acknowledgement}

The study was funded by National Natural Science Foundation of China under 20477010 and 20477054. The author also acknowledge the State Key Laboratory of Environmental Aquatic Chemistry for providing the funding for this research (52TS200801).

\section{References}

Bache, D.H., Johnson, C., McGilligan, J.F., Rasool, E., 1997. A conceptual view of floc structure in the sweep floc domain. Water Sci. Technol. 36, 49-56.

Boller, M., Blaser, S., 1998. Particle under stress. Water Sci. Technol. 37, 9-29.

Bushell, G.C., Yan, Y.D., Woodfield, D., Raper, J., Amal, R., 2002. On techniques for the measurement of the mass fractal dimension of aggregates. Adv. Colloid Interface 95 , $1-50$.

Chakraborti, R.K., Atkinson, J.F., Van Benschoten, J.E., 2000. Characterization of alum floc by image analysis. Environ. Sci. Technol. 34, 3969-3976.

Chakraborti, R.K., Gardenr, K.H., Atkinson, J.F., VanBenschoten, J.E., 2003. Changes in fractal dimension during aggregation. Water Res. 37, 873-883.

Fan, A., Turro, N.J., Somasundaran, P., 2000. A study of dual polymer flocculation. Colloids Surface A 162, 141-148.

Francois, S.B., 1987. Strength of aluminum hydroxide flocs. Water Res. 21, 1023-1030.

Glover, S.M., Yan, Y.D., Jameson, G.J., Biggs, S., 2000. Bridging flocculation studied by light scattering and settling. Chem. Eng. J. 80, 3-12.

Glover, S.M., Yan, Y.D., Jameson, G.J., Biggs, S., 2001. Polymer molecular weight and mixing effects on floc compressibility and filterability. 6th World Congress of Chemical Engineering Melbourne, pp. 23-27.

Gregory, J., 1997. The density of particle aggregates. Water Sci. Technol. 36 (4), 1-13.

Jarvis, P., Jefferson, B., Gregory, J., Parsons, S.A., 2005a. A review of floc strength and breakage. Water Res. 39, 3121-3137.

Jarvis, P., Jefferson, B., Parsons, S.A., 2005b. Breakage, regrowth, and fractal nature of natural organic matter flocs. Environ. Sci. Technol. 39, 2307-2314.

Jarvis, P., Jefferson, B., Parsons, S.A., 2005c. How the natural organic matter to coagulant ratio impacts on floc structural properties. Environ. Sci. Technol. 39, 8919-8924.

Jiang, Q., Logan, B.E., 1991. Fractal dimensions of aggregates determined from steady state size distributions. Environ. Sci. Technol. 25, 2031-2038.

Johnson, C.P., Li, X.Y., Logan, B.E., 1996. Settling velocities of fractal aggregates. Environ. Sci. Technol. 30, 1911-1918.

Koen, G., Willy, V., 1997. Image analysis to estimate the settleability and concentration of activated sludge. Water Res. 31, 1126-1134.

Leu, R.J., Ghosh, M.M., 1988. Polyelectrolyte characteristics and flocculation. J. Am. Water Works Assoc. 80 (4), 159-167.

Li, T., Zhu, Z., Wang, D.S., Yao, C.H., Tang, H.X., 2006. Characterization of floc size, strength and structure under various coagulation mechanisms. Powder Technol. $168,104-110$.

Li, T., Zhu, Z., Wang, D.S., Yao, C.H., Tang, H.X., 2007. The strength and fractal dimension characteristics of alum-kaolin flocs. Int. J. Miner. Process. 82, 23-29.

Nasser, M.S., James, A.E., 2006. The effect of polyacrylamide charge density and molecular weight on the flocculation and sedimentation behaviour of kaolinite suspensions. Sep. Purif. Technol. 52, 241-252.

Negro, C., Blanco, A., Fuente, E., Sanchez, L.M., Tijero, J., 2005. Influence of flocculant molecular weight and anionic charge on flocculation behaviour and on the manufacture of fibre cement composites by the Hatschek process. Cem. Concr. Res. $35,2095-2103$

Ray, D.T., Hogg, R., 1987. Agglomerate breakage in polymer-flocculated suspensions. J. Colloid Interface Sci. 116, 256-268.

Spicer, P.T., Pratsinis, S.E., 1996. Shear-induced flocculation: the evolution of floc structure and the shape of the size distribution at steady state. Water Res. 30, 1049-1056

Tambo, N., Hozumi, H., 1979. Physical characteristics of flocs-II. Water Res. 13, 421-427.

Tambo, N., Watanabe, Y., 1979. Physical characteristics of flocs-I. Water Res. 13, 409-419.

Tang, P., Greenwood, J., Raper, J.A., 2002. A model to describe the settling behavior of fractal aggregates. J. Colloid Interface Sci. 247, 210-219.

Wu, R.M., Lee, D.J., Waite, T.D., Guan, J., 2002. Multilevel structure of sludge flocs. J. Colloid Interface Sci. 252, 383-392.

Yan, Y.D., Glover, S.M., Jameson, G.J., Biggs, S., 2004. The flocculation efficiency of polydisperse polymer flocculants. Int. J. Miner. Process. 73, 161-175.

Ye, C.Q., Wang, D.S., Shi, B.Y., Yu, J.F., Qu, J.H., Edwards, M., Tang, H.X., 2007. Alkalinity effect of coagulation with polyaluminum chlorides: role of electrostatic patch. Colloids Surf. A. 294, 163-173.

Yeung, A.K., Pelton, R., 1996. Micromechanics: a new approach to studying the strength and breakup of flocs. J. Colloid Interface Sci. 184, 579-585.

Yoon, S.Y., Deng, Y.L., 2004. Flocculation and reflocculation of clay suspension by different polymer systems under turbulent conditions. J. Colloid Interface Sci. 278, 139-145.

Yu, X., Somasundaran, P., 1996. Role of polymer conformation in interparticle-bridging dominated flocculation. J. Colloid Interface Sci. 177, 283-287.

Yu, J.F., Wang, D.S., Ge, X.P., Yan, M.Q., Yang, M., 2006. Flocculation of kaolin particles by two typical polyelectrolytes: a comparative study on the kinetics and floc structures. Colloids Surface A 290, 288-294.

Yukselen, M.A., Gregory, J., 2004. The reversibility of floc breakage. Int. J. Miner. Process. 73, 251-259.

Zhao, Y.Q., 2004. Settling behavior of polymer flocculated water-treatment sludge II: effects of floc structure and floc packing. Sep. Purif. Technol. 35, 175-183.

Zhou, Y., Franks, G.V., 2006. Flocculation mechanism induced by cationic polymers investigated by light scattering. Langmuir 22, 6775-6786. 